\title{
Unconventional High Temperature Superconductivity in Cubic Zinc-blende Transition Metal Compounds
}

\author{
Qiang Zhang, ${ }^{1}$ Kun Jiang, ${ }^{1,2}$ Yuhao $\mathrm{Gu},{ }^{1,3}$ and Jiangping $\mathrm{Hu}^{1,4, *}$ \\ ${ }^{1}$ Beijing National Laboratory for Condensed Matter Physics, \\ and Institute of Physics, Chinese Academy of Sciences, Beijing 100190, China \\ ${ }^{2}$ Department of Physics, Boston College, Chestnut Hill, MA 02467, USA \\ ${ }^{3}$ Beijing National Laboratory for Molecular Sciences, State Key Laboratory of Rare Earth \\ Materials Chemistry and Applications, Institute of Theoretical and Computational Chemistry, \\ College of Chemistry and Molecular Engineering, Peking University, 100871 Beijing, China \\ ${ }^{4}$ Kavli Institute of Theoretical Sciences, University of Chinese Academy of Sciences, Beijing, 100190, China

\begin{abstract}
We consider possible high temperature superconductivity (high- $T_{c}$ ) in transition metal compounds with a cubic zinc-blende lattice structure. When the electron filling configuration in the d-shell is close to $d^{7}$, all three $T_{2 g}$ orbitals are near half filling with strong nearest neighbor antiferromagnetic (AFM) superexchange interactions. We argue that upon doping, this electronic environment can be one of "genes" to host unconventional high $T_{c}$ with a time reversal symmetry broken $d_{2 z^{2}-x^{2}-y^{2}} \pm i d_{x^{2}-y^{2}}$ pairing symmetry. With gapless nodal points along the diagonal directions, this state is a direct three-dimensional analogue to the two-dimensional $B_{1 g}$ d-wave state in cuprates. We suggest that such a case may be realized in electron doped CoN, such as $\mathrm{CoN}_{1-x} \mathrm{O}_{x}$ and $(\mathrm{H}, \mathrm{Li})_{1-x} \mathrm{CoN}$.
\end{abstract}

The superconducting mechanism of unconventional high$T_{c}$ in cuprates[1] and iron-based superconductors[2] remains one of the most challenging problems in condensed matter physics[3-5]. A correct answer to this problem should be able to guide us to identify or predict new materials with potential high- $T_{c}$.

Recently, we have identified that a key character, called the electronic gene, which separates these two classes of high- $T_{c}$ materials from other transitional metal compounds, is that the d-orbitals with the strongest in-plane d-p couplings in both high- $T_{c}$ families are isolated near Fermi energy[6-8]. The gene can only be realized by a specific collaboration through cation-anion complexes, global lattice structures, and specific electron filling configurations in the d-shell of transition metal atoms. In cuprates, the $\mathrm{d}_{x^{2}-y^{2}} \mathrm{e}_{g}$ orbital is isolated near Fermi energy in a two-dimensional (2D) $\mathrm{Cu}-\mathrm{O}$ square lattice formed by corner-shared $\mathrm{CuO}_{6}$ octahedra (or $\mathrm{CuO}_{4}$ square planar) in a $d^{9}$ filling configuration of $\mathrm{Cu}^{2+}$. In iron-based superconductors, there are two $T_{2 g} \mathrm{~d}_{x y}$-type orbitals which are isolated near Fermi energy with the $d^{6}$ filling configuration of $\mathrm{Fe}^{2+}$ in a $\mathrm{Fe}(\mathrm{Se} / \mathrm{As}) 2 \mathrm{D}$ square lattice formed by edge-shared $\mathrm{Fe}(\mathrm{Se} / \mathrm{As})_{4}$ tetrahedra[6, 7, 9].

In order to justify the above idea, we must discover new families of high- $T_{c}$. We have suggested to realize the gene condition in the $d^{7}$ and $d^{8}$ filling configurations. Up to now, we have predicted that the $d^{7}$ gene condition can be realized in a 2D hexagonal layer formed by edge-shared trigonal bipyramidal complexes[6] or in a 2D square lattice formed by the corner-shared tetrahedra[10-12], and the $d^{8}$ gene condition exists in a 2D square lattice formed by Ni-based mix-anion octahedra[13]. Unfortunately, all these proposals have not been materialized.

To realize the gene condition, it is easy to notice that a quasi-2D layer is generally required because of the nature of

*Electronic address: jphu@iphy.ac.cn the d-orbital spacial configuration. In fact, all above examples are quasi-2D. However, there may be one exception. In the $d^{7}$ filling configuration with corner shared tetrahedra, all three $T_{2 g}$ orbitals can participate in superconducting pairing. These three orbitals together form a three-dimensional (3D) irreducible representation in a cubic lattice structure. Therefore, even in a $3 \mathrm{D}$ cubic lattice structure, they can be fully isolated near Fermi energy.

The zinc-blende lattice structure which is formed by corner shared tetrahedra is an ideal lattice structure to test this potential exception. Different from the previous examples and proposals, the lattice of the shared tetrahedra in the zinc-blende structure is essentially 3D. Locally, the tetrahedra symmetry is fully respected. The three $T_{2 g}$ orbitals are degenerate and have kinematics in all three spacial directions. If we consider a transition metal compound with the zinc-blende structure, the transition metal atoms form a face center cubic (FCC) lattice as shown in Fig.1(a). Antiferromagnetic superexchange couplings can be generated through anions among all three $T_{2 g}$ d-orbitals due to the crystal field energy splitting in a tetrahedron complex as shown in Fig.1(b). Thus, in the first-order approximation, such an electronic environment can be described by a 3D t-J model.

In this paper, we study the possible superconducting state of a t-J model in a 3D FCC lattice. Both one-orbital and threeorbital models are considered. In both cases, we find that a $d_{2 z^{2}-x^{2}-y^{2}} \pm i d_{x^{2}-y^{2}}$ pairing superconducting state, which breaks the time reversal symmetry, is the most favored near half filling upon hole doping. This $d \pm i d$ state is a spacial 3D analogue to the $2 \mathrm{D} B_{1 g}$ d-wave state in cuprates. Similarly, the gap function vanishes along the diagonal lines, resulting in nodal points in the superconducting states if Fermi surfaces insect with the lines. The extended s-wave state is the least favored followed by chiral t-wave states. We suggest that the case can be potentially realized in electron doped CoN[14], such as $\mathrm{CoN}_{1-x} \mathrm{O}_{x}[15,16]$ and $(\mathrm{H}, \mathrm{Li})_{1-x} \mathrm{CoN}[17,18]$.

We first consider a one-orbital t-J model in a FCC lattice. We assume that the effective hopping between the d-orbitals is 
mainly through d-p couplings and is dominated by the nearest neighbor(NN) hopping. As we will show later, if we ignore the inter-orbital hopping, the kinematics of each $T_{2 g}$ orbital in the zinc-blende lattice is close to that of an isotropic sorbital. Therefore, for simplicity, we start with an isotropic single-orbital model. By setting the second NN distance as one, the kinetic part of the Hamiltonian can be written as $H_{t}=\sum_{k \sigma} a_{k \sigma}^{\dagger} H_{k} a_{k \sigma}$ with

$$
H_{k}=4 t\left(c_{x} c_{y}+c_{z} c_{y}+c_{y} c_{z}\right)-\mu
$$

where $c_{\alpha} \equiv \cos \left(\frac{k_{\alpha}}{2}\right), \alpha=x, y, z$ and $\sigma$ is the spin index. Here $t$ is the nearest neighbor hopping amplitude and $\mu$ is the chemical potential, with $\frac{\mu}{t}=-0.92$ at half filling. The band structure along the high symmetry momenta is shown in Fig.1(c). Assuming the NN AFM superexchange coupling $J$, the total Hamiltonian for a standard $\mathrm{t}-\mathrm{J}$ model can be written as

$$
H=\hat{P}\left(H_{t}\right)+J \sum_{<i j>}\left(S_{i} S_{j}-\frac{1}{4} n_{i} n_{j}\right)
$$

where $\hat{P}$ is the projection operator to forbid double occupancy at a single site and $S_{i}\left(n_{i}\right)$ denotes the spin (density) at site- $i$.

(a)

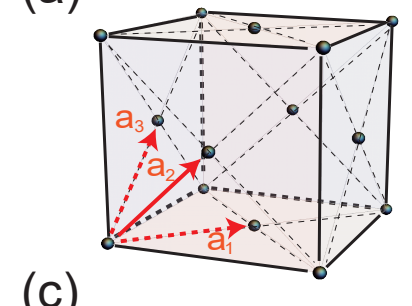

(b)

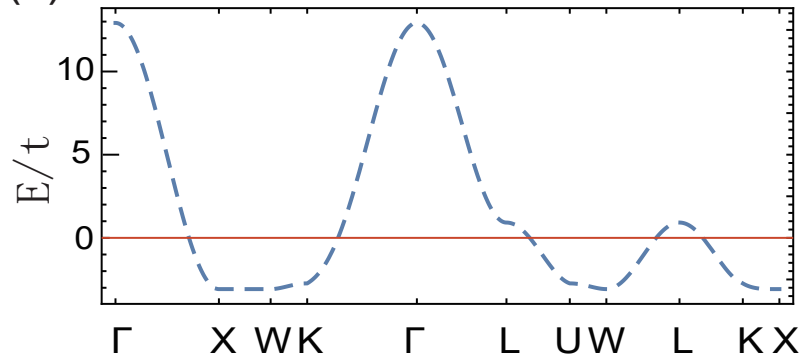

FIG. 1: (a) The face center cubic structure with sketched lattice vectors. (b) The tetrahedra crystal field splitting of the five $3 d$ orbitals. (e) The energy dispersion of the single band model along high symmetry lines.

In a mean field solution, the above Hamiltonian in 2D is known to result in a phase diagram between a long range AFM state and a superconducting state[19], which was known to be qualitatively consistent with experimental results in cuprates[19-21] and iron-based superconductors[22-24]. There is no much difference for this result in 3D space[25]. Moreover, without doping, the antiferromagnetism and magnetic frustration in the FCC structure have already been extensively studied in literature[26-29]. Therefore, we ignore the
AFM phase and solely focus on the superconducting state of the above model upon doping in this paper.

Upon doping, the superconducting state of the above 3D model is more interesting because the lattice symmetry is much larger in three dimensions than in two dimensions. The FCC lattice is governed by the $O_{h}$ point group. The superconducting pairing functions belong to the irreducible representations of the $O_{h}$ group. As the superconducting pairing is induced by the NN AFM interaction, the superconducting order must be in the spin-singlet pairing channel and carry specific momentum form factors. Considering the equal pairing strength for all NN bonds, we have

$$
\begin{aligned}
& \Delta^{A}=4 \delta_{s}\left(c_{x} c_{y}+c_{y} c_{z}+c_{x} c_{z}\right), \\
& \Delta^{E_{ \pm}}=2 \delta_{d}\left(2 c_{x} c_{y}-c_{x} c_{z}-c_{y} c_{z} \pm i \sqrt{3}\left(c_{y} c_{z}-c_{x} c_{z}\right)(4)\right. \\
& \Delta^{T}=-4 \delta_{t}\left(s_{x} s_{y}+e^{i \theta_{1}} s_{y} s_{z}+e^{i \theta_{2}} s_{x} s_{z}\right)
\end{aligned}
$$

where $s_{\alpha}=\sin \left(\frac{k_{\alpha}}{2}\right) . \Delta^{A}$ represents the extended s-wave, $\Delta^{E_{ \pm}}$represents a time reversal symmetry broken superconducting state $d_{2 z^{2}-x^{2}-y^{2}} \pm i d_{x^{2}-y^{2}}$ that belongs to a $2 \mathrm{D} E_{g}$ irreducible representation. It can also be written symmetrically as $4 \delta_{d}\left(c_{x} c_{y}+e^{ \pm i 2 \pi / 3} c_{x} c_{z}+e^{ \pm i 4 \pi / 3} c_{y} c_{z}\right)$. Obviously, this $d \pm i d$ gap function vanishes along the diagonal lines $\left|k_{x}\right|=\left|k_{y}\right|=\left|k_{z}\right|$, as a 3D analogue of the 2D $B_{1 g}$ d-wave for the cuprates. However, in this one-orbital model, near half filling upon hole doping, there is no Fermi surface along the $\Gamma-L$ lines as depicted in Fig.1(c). Therefore the state is still fully gapped. $\Delta^{T}$ represents a t-wave superconducting state that belongs to a $3 \mathrm{D} T_{2 g}$ irreducible representation. In the $\Delta^{T}$ state, besides the pairing strength $\delta_{t}$, we have two additional phase parameters $\theta_{1,2}$. From the standard GinzburgLandau theory analysis[30](see the App.I), we have two types of t-wave phases, the time reversal invariant (TRI) and chiral phases. For the TRI phase, we can take $\theta_{1}=\theta_{2}=0$ and for the chiral one, we can take $\theta_{1}=2 \theta_{2}=4 \pi / 3$.

We use the slave boson mean-field approach[20, 31-33] to calculate the superconducting states in the hole doped region. The results are reported in Fig. 2 with $J / t=0.3$ in (a,b) and $J / t=0.5$ in $(\mathrm{c}, \mathrm{d})$, respectively. The $E_{g}$ state wins over other two states. As shown in Fig.2(a,c), the extended $s$-wave quickly diminishes with doping while the superconducting orders in the $d \pm i d$ wave $E_{g}$ state and the t-wave states appear to be comparable. However, as shown Fig.2(b,d), the $E_{g}$ state has much lower energy than the t-wave states in relevant doping regions. We also notice that the time reversal symmetry broken phases generally gain more condensation energy than the unbroken counterparts. For example, the chiral t-wave state has lower energy than the TRI t-wave state. In Fig.2(e,f), we report the order parameter of the $d \pm i d$ wave state and its condensed energy as the function of the AFM exchange coupling at a fixed doping $x=0.1$.

This result can be easily understood by the Hu-Ding principle[34], which states the favored pairing symmetry is determined by the overlap strength between the momentum form factors of pairing functions and Fermi surfaces. For the extended s-wave, the d-wave and the t-wave states, the form factors peak at $\Gamma, X$ and $L$ high symmetry points, respectively. Moreover, the d-wave can open bigger gaps near $X$, 


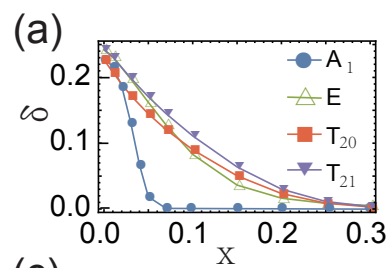

(c)
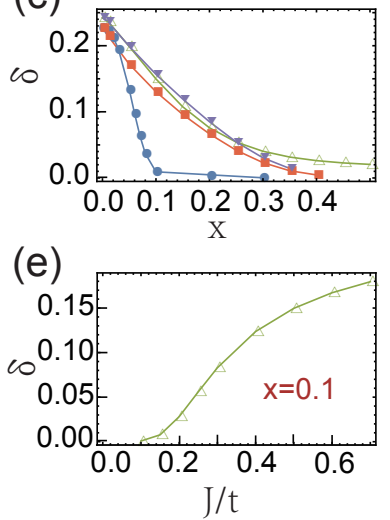

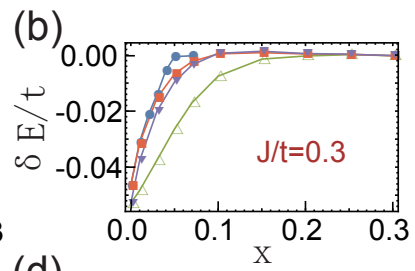

(d)

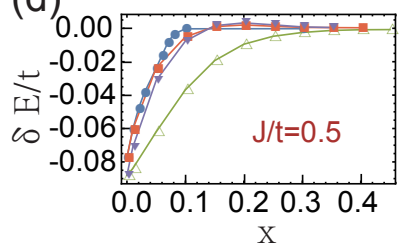

(f)

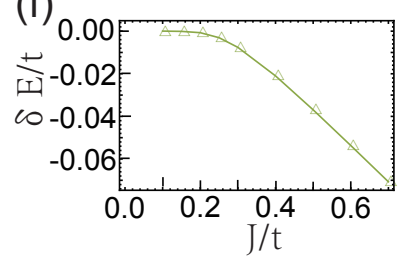

FIG. 2: The results from the slave boson mean field solution of the single-orbital t-J model: (a,c) the pairing strength $\delta_{s / d / t}$ and $(\mathrm{b}, \mathrm{d})$ the ground state energy $\delta E$ with respect to the normal state versus hole doping at $\mathrm{J} / \mathrm{t}=(0.3,0.5)$; here $T_{20}$ and $T_{21}$ denote the TRI and chiral $t$-wave pairing states, respectively. (e, f) the order parameter $\delta_{d}$ and the ground state energy as a function of $\mathrm{J} / \mathrm{t}$ with a hole doping level $x=0.1$.

$W$ and $K$ points. Thus from the energy dispersion in Fig.1(c), the d-wave state wins near half filling upon hole doping. Yet, with heavy electron doping to reach the $L$ (saddle) points, the t-wave can eventually become favored.

Now we consider all three $T_{2 g}$ orbitals. In this case, it is important to note that due to the lack of inversion symmetry, zinc-blende structure belongs to the space group $F \overline{4} 3 m(N o .216)$ and its point group is $T_{d}$ instead of the $O_{h}$ group, as depicted in Fig.3(a). This symmetry difference allows additional hopping terms.

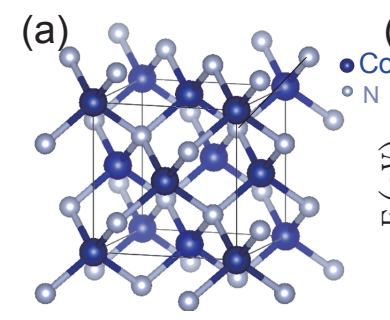

(b)

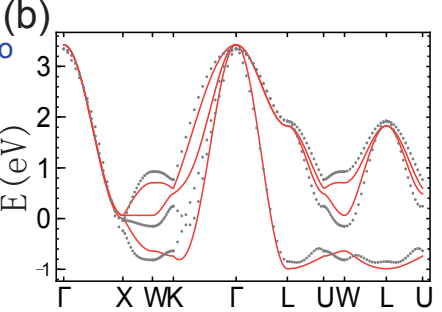

FIG. 3: The zinc-blende structure for $\mathrm{CoN}$ (a) and its three-band dispersion along the high symmetry momenta (b). The NN bond hopping tight-binding dispersion (solid red) with $\left(t_{1}, t_{2}, t_{3}, t_{4}, \mu\right)=$ $(0.214,0.206,0.235,0.084,-0.889) \mathrm{eV}$ fits fairly well with the DFT result (dotted gray).

Taking the cobalt nitrogen (CoN[14]) as an example which has the zinc-blende structure with a lattice constant $4.27 \AA$ as depicted in Fig.3(a), its band structure from density function

theory (DFT[35-37]) is shown in Fig.3(b) with the dotted gray line [46]. The half-filled $T_{2 g}$ orbital is achieved if we dope one more electron per Co atom into this material.

We simplify the band structure in a tight binding model with only the NN hopping among three orbitals. We find that the model is a very good approximation to describe the band dispersion as indicated by the red solid lines in Fig.3(b). To be specific, we define the hopping matrix along $\mathbf{a}_{1}=(1,0,1) / 2$ directions $T\left(\mathbf{a}_{1}\right)$ in the basis $\psi^{\dagger}(\mathbf{r})=\left(d_{y z}^{\dagger}, d_{x z}^{\dagger}, d_{x y}^{\dagger}\right)$. Due to the time reversal symmetry, the mirror symmetry on the plane (110) and the two-fold rotation symmetry along $z$-axis, there are only four free hopping parameters:

$$
T\left(\mathbf{a}_{1}\right)=\left(\begin{array}{ccc}
t_{1} & t_{3} & t_{4} \\
t_{3} & t_{1} & t_{4} \\
-t_{4} & -t_{4} & t_{2}
\end{array}\right),
$$

with $t_{1,2,3,4}$ being real numbers. The hopping matrix along other directions can be obtained by symmetry operations: $T\left(R_{g} \mathbf{a}_{1}\right)=R_{g} T\left(\mathbf{a}_{1}\right) R_{g}^{-1}$. The three-orbital tight-binding Hamiltonian matrix can be written as

$$
H_{3 t}(\mathbf{k})=\sum_{\langle g\rangle} T\left(R_{g} \mathbf{a}_{1}\right) e^{i \mathbf{k} \cdot R_{g} \mathbf{a}_{1}}-\mu,
$$

here $\langle g\rangle \in T_{d}$ ensures that $R_{g} \mathbf{a}_{1}$ runs over all the $12 \mathrm{NN}$ vectors. Specifically, the matrix elements in $H_{3 t}(\mathbf{k})$ are

$$
\begin{aligned}
& H_{3 t, 11}(\mathbf{k})=4\left(t_{1}\left(c_{x} c_{y}+c_{x} c_{z}\right)+t_{2} c_{y} c_{z}\right)-\mu, \\
& H_{3 t, 12}(\mathbf{k})=4\left(i t_{4}\left(c_{x}-c_{y}\right) s_{z}-t_{3} s_{x} s_{y}\right)
\end{aligned}
$$

and other terms can be obtained by cyclical permutation of the suffixes $x, y, z$. For CoN, we find that a set of the parameters with $\left(t_{1}, t_{2}, t_{3}, t_{4}, \mu\right)=$ $(0.214,0.206,0.235,0.084,-0.889) \mathrm{eV}$ fits reasonably well to the DFT calculations as shown Fig.3(b). We notice that $t_{1} \approx t_{2}$, which indicates that the band structure of the threeorbital model is similar to the single-orbital model if the interorbital hoppings are ignored. The interorbital hoppings only cause band splitting around $L$ and $W$ points. The $L$ points splitting stems from the interorbital hopping $t_{3}$ term and the $W$ points splitting comes from the $t_{4}$ term. The small $t_{4}$ term stems from the absence of the inversion symmetry due to the existence of nitrogen atoms (see the App.II). The above band structure is rather qualitatively generic to $T_{2 g} \mathrm{~d}$ orbitals in the zinc-blende lattice structure. One can easily check that with the effective hoppings between the d-orbitals being mainly induced by the d-p couplings, the lattice symmetry qualitatively provides such a band structure.

By adding the AFM exchange interactions, we replace the kinetic energy term in Eq. 2 by $H_{3 t}$ to consider a multi-orbital t-J model[33, 39] and study the possible superconducting states. As we only consider the spin-singlet pairing, the representations of $T_{d}$ group are the same to the even-parity representations of $O_{h}$. Thus, the pairing symmetry analysis for the single-orbital model in FCC structure is applicable to the three-orbital model.

For the superconducting pairing in this multi-orbital t$\mathrm{J}$ model, the intraorbital pairing is always dominant over 
the inter-orbital pairing as shown in previous work[22, 40]. Therefore, we can focus on the superconducting states with only intraorbital pairing. For the three $T_{2 g}$ orbitals, the pairing operators between them can be classified according to the irreducible representations of the $T_{d}$ group as $\hat{T}_{2} \otimes \hat{T}_{2}=$ $\hat{A}_{1} \bigoplus \hat{E} \bigoplus \hat{T}_{1} \bigoplus \hat{T}_{2}$, in which only $\hat{A}_{1}$ and $\hat{E}$ are formed by the intraorbital pairings. Combining with the momentum form factors in Eq.3-5, we can construct the BCS mean field decoupling terms:

$$
\begin{aligned}
& H_{B C S}^{A}=\Delta^{A}(\mathbf{k}) \hat{A}_{1}+\delta_{2}[e(\mathbf{k}) \hat{E}]_{A} \\
& H_{B C S}^{E \pm}=\Delta^{E}(\mathbf{k}) \hat{A}_{1}+\delta_{2}[a(\mathbf{k}) \hat{E}]_{E}+\delta_{3}[e(\mathbf{k}) \hat{E}]_{E} \\
& H_{B C S}^{T}=\Delta^{T}(\mathbf{k}) \hat{A}_{1}+\delta_{2}\left[t_{2}(\mathbf{k}) \hat{E}\right]_{T}
\end{aligned}
$$

with the $\delta_{2,3}$ terms defined in App.III The first terms in all above three pairing equations represent isotropic pairing among all three orbitals. As shown in the inset of Fig.4, in all pairing states, this term is dominant over other pairing terms in our calculations. Thus, in the following paper, we simply focus on the isotropic intraorbital pairings.

Under the slave boson mean-field approach, we study superconducting pairings in the hole doped three-orbital model. Due to the high degeneracy of the three $T_{2 g}$ orbitals, the carrier occupancies in the three orbitals are identical, leading to an equal renormalization factor for all the hopping interactions[33]. The results are reported in Fig.4. Similar to the single-orbital model, the time reversal symmetry broken $d_{2 z^{2}-x^{2}-y^{2}} \pm i d_{x^{2}-y^{2}}$ wave is the most favored state. In this calculation, we take the hopping parameters fitted to $\mathrm{CoN}$ and $J=0.2 \mathrm{eV}$. Following the Hu-Ding principle[34], the $\mathrm{t}$-wave pairing is expected to be weaker in the three-orbital model than in the one-orbital model because of the band splitting at the $L$ points, which is caused by the $t_{3}$ inter-orbital hopping as shown in Fig.3(b). Our calculation confirms this result. Even in the heavy electron doped region, the t-wave never wins over the $d \pm i d$ wave. Moreover, the splitting at $L$ points results in one Fermi surface along the $\Gamma-L$ lines. Thereafter, in the three-orbital model, eight gapless points appear in the $d \pm i d$ wave state, resembling the nodal d-wave state in cuprates. The inset of Fig.4(a) shows that the anisotropic pairing parameter in the second term of the $E$ representation in Eq.10 quickly diminishes with increasing hole doping from half filling. The pairing prefers to maintain equal amplitudes on different orbitals as a result of the high degeneracy of the three $T_{2 g}$ orbitals. This is consistent with the "superconducting fitness" analysis for the multi-orbital pairing[41], as the pairing prefers to take place among the electrons on the same band with the same energy.

In summary, guided by the recent ideas on searching for potential new high temperature superconductors, we studied the superconducting states of a 3D t-J model in both one and three orbital cases under the slave boson meanfield approximation. It was found that the time reversal symmetry broken $d_{2 z^{2}-x^{2}-y^{2}} \pm i d_{x^{2}-y^{2}}$ wave superconducting state wins over all other superconducting states upon hole doping. The state has gapless nodal points along the diagonal directions, being a direct3D extension of the d-wave superconducting state of cuprates. It is also proper to mention that the $3 \mathrm{D} d \pm i d$ pair- (a)

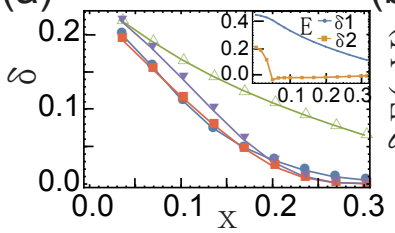

(b)

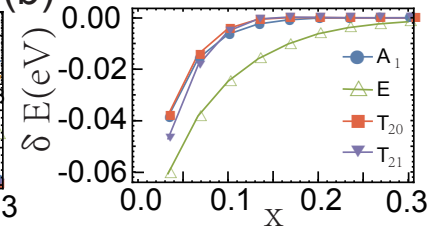

FIG. 4: The slave boson meanfield results for the three-orbital t-J model: (a) the pairing strength $\delta_{s / d / t}$ and (b) the ground state energy versus hole doping away from half-filling. Here the tight-binding parameters for $\mathrm{CoN}$ at Fig. 3 are used and $J=0.2 \mathrm{eV}$. The inset in (a) depicts the intraorbital isotropic $\left(\delta_{1}\right)$ and anisotropic $\left(\delta_{2}\right)$ pairing order parameters for the $E$ representation in Eq.10.

ing symmetry was theoretically suggested previously for the $\beta$-pyrochlore $\mathrm{CsW}_{2} \mathrm{O}_{6}$ [42] by a weak coupling approach[43] without conclusive experimental evidence.

To realize the physics studied in this paper, we must have a zinc-blende transition metal compound with a $d^{7}$ filling configuration at transition metal atoms. As we have mentioned in the paper, $\mathrm{CoN}$ is a material close to a realization of our model. However, as the Co atom in $\mathrm{CoN}$ has valence $\mathrm{Co}^{3+}$, it is a $d^{6}$ filling configuration, namely it is heavily hole doped material with respect to a $T_{2 g}$ half filled $d^{7}$ configuration. Thus, we may consider $\mathrm{CoO}$ with a zinc-blende structure to be the parental compound since the Co atom has valence $\mathrm{Co}^{2+}$ with a $T_{2 g}$ half filled $d^{7}$ configuration. The zinc-blende $\mathrm{CoO}$ was known to be a metastable[44] and have an AFM ground state[27, 45]. The CoN was experimentally known to be a very good metal[16]. Therefore, assuming that the zincblende structure is not drastically modified locally, we can suggest that the potential superconductivity may be realized in $\mathrm{CoO}_{1-x} \mathrm{~N}_{x}$ [15] in which $\mathrm{x}$ describes the concentration of doped hole carriers. Under the same assumption, we may also consider materials by adding electron carriers to $\mathrm{CoN}$, such as $(\mathrm{H}, \mathrm{Li})_{1-x} \mathrm{CoN}[17,18]$, to realize potential superconducting states.

The model has very high symmetry in both orbital and lattice spaces. Here we only consider the superconducting states with respect to the full lattice symmetry. In principle, the degeneracy among three orbitals and the cubic lattice symmetry can be broken spontaneously in many different ways by a variety of mechanisms. Therefore, it can be extremely interesting to explore novel electronic states in future.

Acknowledgement: Q. Zhang acknowledges the support from the International Young Scientist Fellowship of Institute of Physics CAS (Grant No. 2017002) and the Postdoctoral International Program from China Postdoctoral Science Foundation (Grant No. Y8BK131T61). Y. Gu is supported by the High-performance Computing Platform of Peking University. JP. Hu is supported by the Ministry of Science and Technology of China 973 program(Grant No. 2015CB921300, No. 2017YFA0303100), National Science Foundation of China (Grant No. NSFC-11334012), and the Strategic Priority Research Program of CAS (Grant No. XDB07000000). 


\section{LANDAU THEORY FOR PAIRING SYMMETRIES}

The pairing form can be a linear combination of the basis function of a representation $\Delta(\mathbf{k})=\sum_{i} \delta_{i} f_{i}(\mathbf{k})$ with $f_{i}(\mathbf{k})$ being the basis functions and $\delta_{i}$ being the pairing amplitude. In our consideration, the electron pairing takes place on the NN bond mediated by AFM superexchange. Considering an equal on-bond pairing amplitude, as mentioned in the main text, the pairing belongs to the $E$ representation is fixed as $\delta_{d}\left(e_{1} \pm i e_{2}\right)$. However, for the $T_{2}$ representation, $\Delta^{T}=\delta_{x} s_{z} s_{y}+\delta_{y} s_{x} s_{z}+\delta_{z} s_{x} s_{y}$, there is additional phase freedom among the $\delta_{i}$ 's.

We now use Ginzburg-Landau theory to explore the phase freedom of $T_{2}$ representation. The free energy respects all the symmetries including point group, time reversal, $U(1)$ gauge symmetry and spin rotational $S U(2)$ symmetry[30]. The superconducting phases are determined by the fourth order terms. We extract the invariant $A_{1}$ forms from $T_{2} \otimes T_{2}^{*} \otimes$ $T_{2} \otimes T_{2}^{*}$. They are

$$
\begin{aligned}
& -\left(\left|\delta_{x}\right|^{2}+\left|\delta_{y}\right|^{2}+\left|\delta_{z}\right|^{2}\right)^{2}, \\
& \text { - }\left|\delta_{x}\right|^{4}+\left|\delta_{z}\right|^{4}+\left|\delta_{z}\right|^{4}-\left(\left|\delta_{y} \delta_{z}\right|^{2}+\left|\delta_{x} \delta_{z}\right|^{2}+\left|\delta_{y} \delta_{x}\right|^{2}\right), \\
& \text { - }\left(\delta_{x} \delta_{y}^{*} \pm \delta_{x}^{*} \delta_{y}\right)^{2}+\left(\delta_{x} \delta_{z}^{*} \pm \delta_{x}^{*} \delta_{z}\right)^{2}+\left(\delta_{z} \delta_{y}^{*} \pm \delta_{x}^{*} \delta_{z}\right)^{2} .
\end{aligned}
$$

Those are essentially the polynomial invariants as we project $\left|\delta_{x}+\delta_{y}+\delta_{z}\right|^{4}$ on the basis functions. Only three of them are independent and they can be rephrased with three independent parameters in the fourth order term:

$$
\begin{aligned}
\Delta F_{4}(\boldsymbol{\delta})= & \beta_{1}\left(\left|\delta_{x}\right|^{2}+\left|\delta_{y}\right|^{2}+\left|\delta_{z}\right|^{2}\right)^{2}+\beta_{2}\left(\left|\delta_{y} \delta_{z}\right|^{2}+\left|\delta_{x} \delta_{z}\right|^{2}\right. \\
& \left.+\left|\delta_{y} \delta_{x}\right|^{2}\right)+\beta_{3}\left|\delta_{x}^{2}+\delta_{y}^{2}+\delta_{z}^{2}\right|^{2}
\end{aligned}
$$

Considering the equal NN bonds pairing amplitude $\delta=$ $\delta_{t}\left(1, e^{i \theta_{1}}, e^{i \theta_{2}}\right)$, mediated by the NN AFM exchange interactions, the free energy becomes

$$
\Delta F_{4}(\boldsymbol{\delta})=\delta_{t}^{4}\left(9 \beta_{1}+3 \beta_{2}+\beta_{3}\left|1+e^{i 2 \theta_{1}}+e^{i 2 \theta_{2}}\right|^{2}\right) .
$$

The minimization of $\Delta F_{4}(\boldsymbol{\delta})$ gives the following phases characterized by $\left(\theta_{1}, \theta_{2}\right)$ :

- TRI states: $\beta_{3}<0$ and $3 \beta_{1}+\beta_{2}+3 \beta_{3}>0$

- ferro: $(0,0)$

- antiferro: $(0, \pi)$ or $(\pi, 0)$ or $(\pi, \pi)$;

- Chiral states: $\beta_{3}>0$ and $3 \beta_{1}+\beta_{2}>0$

$$
\begin{aligned}
& \text { - }(4 \pi, 2 \pi) / 3 \text { or }(2 \pi, 4 \pi) / 3 \\
& -(4 \pi, 5 \pi) / 3 \text { or }(5 \pi, 4 \pi) / 3 \\
& -(\pi, 2 \pi) / 3 \text { or }(2 \pi, \pi) / 3 \\
& -(\pi, 5 \pi) / 3 \text { or }(5 \pi, \pi) / 3
\end{aligned}
$$

The TRI state $s_{z} s_{y} \pm s_{x} s_{z} \pm s_{x} s_{y}$ are fourfold degenerate and the chiral state $s_{z} s_{y} \pm \omega s_{x} s_{z} \pm \omega^{*} s_{x} s_{y}$ state are eight-fold degenerate with $\omega=e^{ \pm i 2 \pi / 3}$. The states marked with $T_{20}$ and $T_{21}$ are the states $(0,0)$ and $(4 \pi, 2 \pi) / 3$, respectively.

\section{SYMMETRY ANALYSIS FOR THREE-ORBITAL TIGHT-BINDING MODEL}

For the three-orbital tight-binding model, the Hamiltonian $H=\sum d_{\alpha \mathbf{k}}^{\dagger} H_{3 t, \alpha \beta}(\mathbf{k}) d_{\beta \mathbf{k}}$ shall be the $A_{1}$ representation of the point group. It contains the orbital parts and the momentum factor parts. The orbital parts are the representation product and it can be decomposed as : $\hat{T}_{2} \otimes \hat{T}_{2}=$ $\hat{A}_{1} \bigoplus \hat{E} \bigoplus \hat{T}_{1} \bigoplus \hat{T}_{2}$. To make the Hamiltonian $A_{1}$ representation, the factor parts should be the same representations with the orbital parts. Firstly considering the intraorbital hopping terms, the orbital parts can form $\hat{A}_{1}$ representation as $d_{y z}^{\dagger} d_{y z}+d_{x z}^{\dagger} d_{x z}+d_{x y}^{\dagger} d_{x y}$ and $\hat{E}$ representation as $\left(2 d_{x y}^{\dagger} d_{x y}-d_{y z}^{\dagger} d_{y z}-d_{x z}^{\dagger} d_{x z}, d_{y z}^{\dagger} d_{y z}-d_{x z}^{\dagger} d_{x z}\right)$. Combining with the factor of $A_{1}$ and $E$ forms $\left(e_{1}, e_{2}\right)$, they can be rephrased as the $t_{1}$ and $t_{2}$ terms in the Hamiltonian. For the inter-orbital hopping, the $\hat{T}_{2}$ representation basis can be written as $\left(d_{x z}^{\dagger} d_{x y}, d_{y z}^{\dagger} d_{x y}, d_{y z}^{\dagger} d_{x z}\right)+h . c$. , with h.c. denoting the Hermitian conjugate counterparts. Combining with the factors $\left(s_{y} s_{z}, s_{x} s_{z}, s_{x} s_{y}\right)$, they are the $t_{3}$ terms. The above terms do not break inversion symmetry so that $t_{1,2,3}$ are real numbers. Due to the existence of nitrogen atoms, the $O_{h}$ group reduces to $T_{d}$ for the breach of inversion symmetry. This allows us to write down the $t_{4}$ term which breaks inversion symmetry. They come from the combination of $\hat{T}_{1}$ representation of the orbitals $i\left(d_{x z}^{\dagger} d_{x y}, d_{y z}^{\dagger} d_{x y}, d_{y z}^{\dagger} d_{x z}\right)+h . c$ and the factors $\left(s_{x}\left(c_{y}-c_{z}\right), s_{y}\left(c_{z}-c_{x}\right), s_{z}\left(c_{x}-c_{y}\right)\right)$. From time reversal symmetry and Hermitian condition of the Hamiltonian, $t_{4}$ is real.

\section{THREE-ORBITAL MODEL PAIRING FORMS}

In the pairing part of Eq.(9-11) of the three-orbital model, the orbital quadratic forms $\hat{A}_{1}$ and $\hat{E}$ are:

$$
\begin{aligned}
& \hat{A}_{1}=d_{y z}^{\dagger} d_{y z}^{\dagger}+d_{x z}^{\dagger} d_{x z}^{\dagger}+d_{x y}^{\dagger} d_{x y}^{\dagger} \\
& \hat{E}^{1}=2 d_{x y}^{\dagger} d_{x y}^{\dagger}-d_{x z}^{\dagger} d_{x z}^{\dagger}-d_{y z}^{\dagger} d_{y z}^{\dagger} \\
& \hat{E}^{2}=\sqrt{3}\left(d_{y z}^{\dagger} d_{y z}^{\dagger}-d_{x z}^{\dagger} d_{x z}^{\dagger}\right)
\end{aligned}
$$

where the spin and momentum labels are omitted for the momentum conserved spin-singlet pairing forms. The additional pairing forms of $\delta_{2,3}$ are

$$
\begin{aligned}
{[e(\mathbf{k}) \hat{E}]_{A}=} & \sum_{\sigma= \pm} \Delta^{E_{\sigma}}(\mathbf{k})\left(\hat{E}^{1}-i \sigma \hat{E}^{2}\right) \\
{[a(\mathbf{k}) \hat{E}]_{E}=} & 4\left(c_{x} c_{y}+c_{y} c_{z}+c_{x} c_{z}\right)\left(\hat{E}^{1} \pm i \hat{E}^{2}\right) \\
{[e(\mathbf{k}) \hat{E}]_{E}=} & \left(e_{1} \hat{E}^{1}-e_{2} \hat{E}^{2} \pm i\left(e_{1} \hat{E}^{2}+e_{2} \hat{E}^{1}\right)\right) \\
{\left[t_{2}(\mathbf{k}) \hat{E}\right]_{T}=} & s_{y} s_{z}\left(\hat{E}^{1}-\sqrt{3} \hat{E}^{2}\right)+e^{i \theta_{1}} s_{x} s_{z}\left(\hat{E}^{1}+\sqrt{3} \hat{E}^{2}\right) \\
& +e^{i \theta_{2}} 2 s_{x} s_{y} \hat{E}^{1}
\end{aligned}
$$

with $e_{1}=2 c_{x} c_{y}-c_{x} c_{z}-c_{y} c_{z}$ and $e_{2}=\sqrt{3}\left(c_{y} c_{z}-c_{x} c_{z}\right)$. 
[1] J. G. Bednorz and K. A. Muller, Z. Phys. B 64, 189 (1986).

[2] Y. Kamihara, T. Watanabe, M. Hirano, and H. Hosono, J. Am. Chem. Soc. 130, 3296 (2008).

[3] J. Zaanen, S. Chakravarty, T. Senthil, P. Anderson, P. Lee, J. Schmalian, M. Imada, D. Pines, M. Randeria, C. Varma,et al., Nat. Phys. 2, 138 (2006).

[4] M. R. Norman, Science 332, 196 (2011).

[5] A. J. Leggett, Nat. Phys. 2, 134 (2006).

[6] J. Hu, C. Le, and X. Wu, Phys. Rev. X 5, 041012 (2015).

[7] J. Hu, Sci. Bull. 61, 561 (2016).

[8] J. Hu and J. Yuan, Front. Phys. 11, 117404 (2016).

[9] J. Hu and N. Hao, Phys. Rev. X 2, 021009 (2012).

[10] J. Hu and C. Le, Sci. Bull. 62, 212 (2017).

[11] G. E. Delgado, P. Grima-Gallardo, L. Nieves, H. Cabrera, J. R. Glenn, and J. A. Aitken, Mater. Res. 19, 1423 (2016).

[12] J. Hu, Y. Gu, and C. Le, Sci. Bull. 63, 1338 (2018).

[13] C. Le, J. Zeng, Y. Gu, G.-H. Cao, and J. Hu, Sci. Bull. 63, 957 (2018).

[14] K. Suzuki, T. Kaneko, H. Yoshida, H. Morita, and H. Fujimori, J. Alloy. Comp. 224, 232 (1995).

[15] M.-W. Lumey and R. Dronskowski, Adv. Funct. Mater. 14, 371 (2004).

[16] J. Takahashi, Y. Hirose, D. Oka, S. Nakao, C. Yang, T. Fukumura, I. Harayama, D. Sekiba, and T. Hasegawa, Appl. Phys. Lett. 107, 231906 (2015).

[17] M. Reddy, G. Prithvi, K. P. Loh, and B. Chowdari, Appl. Mater. Interfaces 6, 680 (2013).

[18] H. Wang, X. Song, Y. Xu, and Z. Yang, Mod. Phys. Lett. B 32, 1850184 (2018).

[19] G. Kotliar and J. Liu, Phys. Rev. B 38, 5142 (1988).

[20] J. Brinckmann and P. A. Lee, Phys. Rev. B 65, 014502 (2001).

[21] P. A. Lee, N. Nagaosa, and X.-G. Wen, Rev. Mod. Phys. 78, 17 (2006).

[22] K. Seo, B. A. Bernevig, and J. Hu, Phys. Rev. Lett. 101, 206404 (2008).

[23] R. Yu and Q. Si, Phys. Rev. B 86, 085104 (2012).

[24] Q. Si, E. Abrahams, J. Dai, and J.-X. Zhu, New J. Phys 11, 045001 (2009).

[25] I. Ichinose, T. Matsui, and M. Onoda, Phys. Rev. B 64, 104516 (2001).
[26] J. S. Smart, Phys. Rev. 86, 968 (1952).

[27] W. L. Roth, Phys. Rev. 110, 1333 (1958).

[28] M. E. Lines, Phys. Rev. 139, A1304 (1965).

[29] N.-N. Sun and H.-Y. Wang, J. Magn. Magn. Mater 454, 176 (2018).

[30] M. Sigrist and K. Ueda, Rev. Mod. Phys. 63, 239 (1991).

[31] A. Ruegg, M. Indergand, S. Pilgram, and M. Sigrist, Eur. Phys. J. B 48, 55 (2005).

[32] Q.-H. Wang, D.-H. Lee, and P. A. Lee, Phys. Rev. B 69, 092504 (2004).

[33] Y. Gu, Q. Zhang, C. Le, Y. Li, T. Xiang, and J. Hu, Phys. Rev. B 100, 165405 (2019).

[34] J. Hu and H. Ding, Sci. Rep. 2, 381 (2012).

[35] G. Kresse and J. Furthmuller, Phys. Rev. B 54, 11169 (1996).

[36] G. Kresse and D. Joubert, Phys. Rev. B 59, 1758 (1999).

[37] J. P. Perdew, K. Burke, and M. Ernzerhof, Phys. Rev. Lett. 77, 3865 (1996).

[38] Our DFT calculations employ the VASP code[35] with the PAW method[36] and the PBE[37] exchange-correlation functional.The kinetic energy cutoff of $600 \mathrm{eV}$ for the planewaves, $\Gamma$-centered k-mesh of $16 \times 16 \times 16$ and energy convergence criterion of $10^{-6} \mathrm{eV}$ are adopted.

[39] K. Jiang, X. Wu, J. Hu, and Z. Wang, Phys. Rev. Lett. 121, 227002 (2018).

[40] Z. Yin, K. Haule, and G. Kotliar, Nat. Phys. 10, 845 (2014).

[41] A. Ramires, D. F. Agterberg, and M. Sigrist, Phys. Rev. B 98, 024501 (2018).

[42] S. V. Streltsov, I. I. Mazin, R. Heid, and K.-P. Bohnen, Phys. Rev. B 94, 241101 (2016).

[43] D. F. Agterberg, V. Barzykin, and L. P. Gorkov, Phys. Rev. B 60, 14868 (1999).

[44] M. Redman and E. Steward, Nature 193, 867 (1962).

[45] H.-X. Deng, J. Li, S.-S. Li, J.-B. Xia, A. Walsh, and S.-H. Wei, Appl. Phys. Lett. 96, 162508 (2010).

[46] Our DFT calculations employ the VASP code[35] with the PAW method[36] and the PBE[37] exchange-correlation functional. The kinetic energy cutoff of $600 \mathrm{eV}$ for the planewaves, $\Gamma$ centered k-mesh of $16 \times 16 \times 16$ and energy convergence criterion of $10^{-6} \mathrm{eV}$ are adopted. 